\title{
Development and Validation of a Lncrnas-Based Nomogram for Survival Prediction in Neuroblastoma Patients
}

Yong Lv

Sichuan University West China Hospital

ShuGuang Jin

Sichuan University West China Hospital

Bo Xiang ( $\square$ xb_scu_edu@163.com )

Sichuan University West China Hospital https://orcid.org/0000-0001-9794-6801

\section{Research}

Keywords: Neuroblastoma, Long non-coding RNAs, Nomogram, Prognosis

Posted Date: May 7th, 2021

DOl: https://doi.org/10.21203/rs.3.rs-448031/v1

License: (c) (1) This work is licensed under a Creative Commons Attribution 4.0 International License.

Read Full License 


\section{Abstract}

\section{Background}

Treatment of neuroblastoma is evolving toward precision medicine. LncRNAs can be used as prognostic biomarkers in many types of cancer.

\section{Methods}

Based on the RNA-seq data from GSE49710, we built a IncRNAs-based risk score using the least absolute shrinkage and selection operation (LASSO) regression. Cox regression, receiver operating characteristic curves were used to evaluate the association of the LASSO risk score with overall survival. Nomograms were created and then validated in an external cohort from TARGET database. Gene set enrichment analysis was performed to identify the significantly changed biological pathways.

\section{Results}

The 16-IncRNAs-based LASSO risk score was used to separate patients into high-risk and low-risk groups. In GSE49710 cohort, the high-risk group exhibited a poorer OS than those in the low-risk group $(P<0.001)$. Moreover, multivariate Cox regression analysis demonstrated that LASSO risk score was an independent risk factor ( $\mathrm{HR}=6.201 ; 95 \% \mathrm{Cl}: 2.536-15.16)$. The similar prognostic powers of the 16-IncRNAs were also achieved in the external cohort and in stratified analysis. In addition, a nomogram was established and worked well both in the internal validation cohort $(\mathrm{C}$-index $=0.831)$ and external validation cohort (Cindex $=0.773$ ). The calibration plot indicated the good clinical utility of the nomogram. Gene set enrichment analysis (GSEA) indicated that high-risk group was related with cancer recurrence, metastasis and inflammatory associated pathways.

\section{Conclusion}

The IncRNA-based LASSO risk score is a promising and potential prognostic tool in predicting the survival of patients with neuroblastoma. The nomogram combined the IncRNAs and clinical parameters allows for accurate risk assessment in guiding clinical management.

\section{Introduction}

Neuroblastoma originates from neural stem cells, is one of the most common malignant tumors in children[1]. Risk-based therapeutic methods combined with surgery is an important component of therapies for neuroblastoma, which requires an optimization of overall survival estimation for specific patient[2]. Indeed, patients can be generally classified into low-risk or high-risk groups depending on their tumor MYCN status and International Neuroblastoma Staging System (INSS). However, it does not account for several known prognostic factors (eg, age, sex) and unknown prognostic factors (eg, molecular biomarkers). The conventional prognostic factors such as the INSS stage and MYCN status are insufficient to predict neuroblastoma patient outcome, treatment outcome remains undesirable[3]. 
Due to the high levels of heterogeneity of neuroblastoma, the prognosis remains quite unsatisfactory[4]. Several studies have attempted to integrate multiple biomarkers into a single model and improve specificity and individual risk assessment [5]. The discovery of new reliable molecular biomarkers will guide the prognostic evaluation of neuroblastoma patients. Therefore, a more accurate molecular biomarker for precise prognostic prediction are needed. Currently, with the development of RNAsequencing (RNA-Seq) and bioinformatics, the roles of dysregulated functional long noncoding RNAs (IncRNAs) in human cancers have received considerable attention[6]. The IncRNAs are non-coding RNAs that range in length from 200 nucleotides to over 100 kilobases, which has been identified to serve a critical role in various types of cancer, and some of them have been implicated in diagnosis and prognostication[7]. Although some IncRNAs for neuroblastoma prognosis prediction have been tested in basic medicine trials such as IncRNA SNHG16 [8], IncNB1 [9], FOXD3-AS1[10], more potential and valuable IncRNAs are urgent to be discovered.Integrating multiple IncRNAs into a IncRNA signature model might create predictive and prognostic value in the management of neuroblastoma.

A nomogram is a graphical analogue computation device that can be used to evaluate the prognostic biomarkers in oncology and medicine. Nomograms makes the prediction model readable by generating an individual probability of a clinical event, leading us closer toward personalized medicine[11]. In this study, we conducted a comprehensive analysis of IncRNAs profiling of neuroblastoma based on a bioinformatics method. By using the least absolute shrinkage and selection operation (LASSO) algorithm, a novel IncRNAs signature was built to predict the survival outcomes of patients with neuroblastoma. Furthermore, we constructed a nomogram to accurately predict the prognosis for neuroblastoma patients.

\section{Methods}

\section{Neuroblastoma datasets and differential expression analysis}

A flowchart which depicted the whole process of our analysis was firstly plotted in Figure 1. Raw microarray data from GSE49710 was directly downloaded from Gene Expression Omnibus (GEO) (https://www.ncbi.nlm.nih.gov/geo/). GSE49710 was conducted by GPL16876 Agilent-020382 Human Custom Microarray 44k. The inclusion criterias included: patients pathologically diagnosed with neuroblastoma; without other malignant tumor history; complete follow-up information. Patients were excluded if the clinical information was incomplete. Total 498 neuroblastoma samples with complete follow-up information were obtained. The comprehensive gene annotation for IncRNA was downloaded from the GENCODE (release 32). All the long non-coding RNAs were extracted from GPL16876 for the preliminary screening of prognostic IncRNAs. The approach of IncRNA profile analysis mainly referred to previous study[12]. Finally, 3798 annotated IncRNA transcripts were generated. We used the limma package in R software to identify differentially expressed IncRNAs ( $P$-value $<0.05$ and $\| \log _{2}$ Fold-change (FC) $\mid>1)[13]$.

\section{Construction of the IncRNA-based prognostic signature}


The LASSO can be used to select optimal features in microarray data with a robust prognostic value[14]. After screening out the differentially expressed IncRNAs, we carried out LASSO regression to select the IncRNAs for prediction of the overall survival. The "glmnet" package in R software was used to perform the LASSO regression analysis. Coefficients from LASSO regression were used to construct prognostic signatures. Then the IncRNA-based LASSO risk score of each sample was calculated according to expression levels of the IncRNAs multiply by LASSO coefficients. Finally, a multivariate Cox regression analysis was conducted to assess the contribution of a IncRNA-based LASSO risk score as an independent prognostic factor for neuroblastoma patient overall survival. The optimal cut-off of LASSO risk score was obtained using "survminer" package (https://CRAN.R-project.org/package=survminer) in R. Then the neuroblastoma patients were divide into the high risk group and low risk group based on the median LASSO risk score value. Kaplan-Meier curves were used to compare the two groups regarding their survival outcomes with the assistance of the log-rank test. Receiver operating characteristic (ROC) curve analysis was employed to assess the performance of survival prediction. A $P$-value $<0.05$ was considered significant.

\section{External data validation}

To further verify the predictive value of the IncRNA-based LASSO risk score, another independent dataset from the Therapeutically Applicable Research To Generate Effective Treatments (TARGET) database as the validation series. TARGET Neuroblastoma, a gene expression array in TARGET database that contains the expression values for part of the differentially expressed IncRNAs, as well as prognosis information for 190 neuroblastoma cases, was enrolled as a validation set (https://ucscpublic.xenahubs.net). Survival curves were plotted using the Kaplan-Meier method.

\section{Construction of the nomogram}

A composite nomogram predicting the overall survival of neuroblastoma was generated by the "rms" package(https://CRAN.R-project.org/package=rms) of R statistical software. The nomogram models were internally validated by use of bootstrap with 200 resamples[15]. The concordance index (C-index) was used to estimate predictive performance of the nomogram. Calibration plots were performed to observed and predicted probabilities for the nomogram.

\section{Gene set enrichment analysis}

To elucidate the potential influence of IncRNAs on the neuroblastoma, an enrichment analysis was conducted between the high-risk and low-risk groups using the Java GSEA implementation (http://www.broadinstitute.org/gsea). Data used for GSEA were accessible from GSE49710. The expression profile data of 498 neuroblastoma cases were sorted in terms of IncRNA-based LASSO risk score. Standard settings with 1000 runs of gene permutations were employed. A normalized enrichment score (NES) is calculated for each gene set based on the size of the set. Nominal p-value and FDR qvalue $<0.05$ was chosen as the cut-off criteria[16]. 


\section{Statistical analysis}

The distribution of overall survival was estimated by the Kaplan-Meier method and compared by log-rank test. Cox proportional hazards models were used to estimate hazard ratios and $95 \%$ confidence intervals (Cls). The $P$ value $<0.05$ was considered statistically significant. All statistical tests were performed with R software (Version 3.6.1).

\section{Results}

\section{Identification of prognostic IncRNAs}

We developed a flow diagram to describe our study (Figure 1). A total of 498 neuroblastoma patients with clinical data were downloaded from the GSE49710 dataset. Another 190 neuroblastoma patients with clinical data were downloaded from the TARGET dataset. The details of patient characteristics were listed in Table 1. In the presented study, a total of 151 differentially expressed IncRNAs (111 upregulated and 40 downregulated) were identified. These IncRNAs were further analyzed by LASSO regression algorithm (Figure2). With this method, total 16 IncRNAs with nonzero coefficients were selected for further analysis.

\section{Identification of a multi-IncRNA-based LASSO risk score}

Median follow-up was 4.7 years. A LASSO-risk-score formula was created based on the expression of these 16 IncRNAs for OS prediction, as follows: LASSO risk score $=\left(0.003718718^{\star}\right.$ expression level of AL137003.2) + (0.008798275*expression level of NKAIN3-IT1 $)+(0.021625392 *$ expression level of ZFHX4-AS1) + $(0.05355537 *$ expression level of PCAT7 $)+(0.060417924 *$ expression level of AL356599.1) + $\left(0.06863845^{\star}\right.$ expression level of BX284668.5) + $\left(0.108933478^{*}\right.$ expression level of AC026790.1 $)+(0.115143797 *$ expression level of AC006206.1 $)+\left(0.180433185^{*}\right.$ expression level of MIR7515HG $)+(-0.186462429 *$ expression level of FOXD1-AS1 $)+\left(-0.151477451^{*}\right.$ expression level of AC022075.1 $)+(-0.123587042 *$ expression level of RFPL1S $)+(-0.111751352 *$ expression level of AC087477.2) $+(-0.054095549 *$ expression level of AC008105.3 $)+(-0.014885334 *$ expression level of AC025259.3) + (-0.011738356* expression level of AP000525.1). We then calculated the 16-IncRNAbased LASSO risk score for each patient in the GSE49710, and ranked them according to their LASSO risk score. The median score (0.0071) of the whole series was adopted as the cut-off value. As such, patients were divided into a high-risk group $(n=249)$ or a low-risk group $(n=249)$. Patients in the high-risk group had significantly shorter overall survival than those in the low-risk group (log-rank test $P<0.0001$ ) (Fig3A). ROC curve indicated the LASSO risk score had a comparative ability in predicting overall survival (Fig3B). Further, in the univariate cox regression model, the LASSO risk score was a strong variable correlated with prognosis in validation datasets. After multivariate adjustment by clinical factors, the LASSO risk score and INSS stage remained a powerful and independent prognostic factor (Table2). We stratified patients into different group based on age (< 18 months or $\geq 18$ months), INSS stage (I + II or III + IV + IVs) and MYCN status (amplified or without amplified). These results showed that the 16-IncRNAbased LASSO risk score can still separate neuroblastoma patients into high-risk or low-risk group, and patients in high-risk group have shorter OS and poor prognosis than those in low-risk group (Fig4). 


\section{External validation of the LASSO risk score}

To further assess the predictive prognostic value of the LASSO risk score, total 190 neuroblastoma patients without adjuvant therapy from the TARGET database (external validation series) were used to validate our results. Patients were separated into high-risk and low-risk group based on the LASSO risk score. Patients with high LASSO risk score had a higher mortality rate than those with low LASSO risk score (Fig5A). ROC curve indicated the LASSO risk score had a comparative ability in predicting overall survival(Fig5B). The results from univariate and multivariate COX regression analyses showed that the identified independent risk factors for OS included the LASSO risk score and INSS stage (Table 3).

\section{Nomogram development}

To predict the overall survival probability of patients with neuroblastoma using a quantitative method, we assembled a nomogram that integrated both the 16-IncRNA-based LASSO risk score and various clinical features to predict 5-year overall survival probability (Fig6). The C-index for the prediction nomogram was 0.831 (95\% Cl:0.785-0.873) in the GSE49710 internal validation cohort. The calibration curve indicated that the nomogram had good accuracy as an ideal model (Fig7A). Application of the nomogram in the TARGET external validation cohort still gave good discrimination (C-index $=0.773 ; 95 \% \mathrm{Cl}: 0.701-0.843$ ) and good calibration (Fig7B).

\section{Gene set enrichment analysis}

We performed GSEA to identify correlated biological process and signaling pathways using the 16IncRNA signature on the basis of risk score for classification. Significant gene sets were selected: E2F targets, Inflammatory response, KRAS signal pathway, TNF-a signal pathway. We proposed that the 16IncRNAs might produce a marked effect by influencing these process and pathways (Fig8).

\section{Discussion}

Neuroblastoma is a common tumor in infants and young children. Neuroblastoma is a group of heterogeneous tumors with different prognosis. Clear clinical risk grouping is important for making treatment plan and evaluating prognosis. Precision medicine is a medical model that customizes treatment plans for patients according to their characteristics in genome, phenotype, and biomarkers[17]. Nomogram is a statistical model that can be used for clinical events predictive analysis. Compared with other predictive statistical methods, nomogram can provide better prognostic risk assessment in a visual way. To date, some nomograms have been constructed to predict the prognosis of patients with cancer. Base on the cancer genome atlas database, some researchers developed a nomogram that incorporate an autophagy-related signature for predicting the survival of glioblastoma patients[18]. Another previous study has constructed a nomogram that could be used to predict the prognosis of uterine corpus endometrial carcinoma [19]. In an effort to guide risk-based treatment for patients with neuroblastoma, we developed and validated nomograms that were based on combinations of clinical and molecular 
prognostic markers. The nomogram may help perform risk stratification and provide more individualized clinical advice for each patient.

Recent studies have shown that LncRNA can regulate many important life activities, and it is also closely related to the occurrence and development of tumor [20]. However, the prognostic value of IncRNAs in neuroblastoma is not entirely clear. In this research, we constructed and validated a 16-IncRNA-based LASSO risk score to predict overall survival for patients with neuroblastoma. Patients in the low-risk group had significantly better survival than those in the high-risk group. Our research highlighted the prognostic value of the 16-IncRNAs and suggested practical applications in prognostic prediction of neuroblastoma. The IncRNA FOXD1-AS1 functions as an important oncogenic IncRNA in glioma and affected biological processes via protein elF5a[21].In breast cancer, higher IncRNA ZFHX4-AS1 expression is associated with worse prognosis, and IncRNA ZFHX4-AS1 acts as an oncogene through the Hippo signaling pathway [22].In non-small cell lung cancer, IncRNA PCAT7 can promote cell proliferation and induce epithelial-mesenchymal transition, it has been well documented as a key regulator of tumour growth and development [23]. The biological function of the remaining IncRNAs in our study has not been investigated in previous studies. Further researches are required to explore the underlying molecular mechanisms of these IncRNAs. These data might improve the development of a cheap molecular test. In our study, stratified analysis were performed to further evaluate whether the 16-IncRNA-based LASSO risk score exhibit predictive effect within same clinical characteristics. We stratified patients into different group based on age, INSS stage and MYCN status. These results showed that the 16-IncRNA-based LASSO risk score can still separate neuroblastoma patients into high-risk or low-risk group, and patients in high-risk group have shorter overall survival and poor prognosis than those in low-risk group. These results demonstrated that this 16 -IncRNA-based LASSO risk score was independent risk factors for survival prediction of neuroblastoma patients and could stratify patients from different group into subtypes with different prognosis. Thus, the ability of our 16-IncRNAs in identifying subgroups of neuroblastoma patients implies that the 16-IncRNAs may be used to refining the current prognostic model and promoting further stratification of patients in the future clinical research.

Most of these dysregulated IncRNAs are not yet functionally annotated. However, we can infer the underlying regulatory function of the IncRNAs using the mRNA expression data of the same group of patients. In order to identify a group of pathways significantly enriched in high-risk group with respect to low-risk group, we performed gene set enrichment analysis (GSEA) using the Java GSEA implementation. The neuroblastoma patients in GSE49710 were assigned into two groups (high risk vs. low risk) according to the optimal cut-off of LASSO-risk-score. Here, the 16-IncRNAs identified in our study are involved in biological pathway known to be crucial to the relapse of neuroblastoma, namely KRAS signalling pathway. A study involving 32 samples have reported that KRAS mutations have been found in some cases of relapse neuroblastomas[24]. E2F-mediated associated pathways were significantly associated with the recurrence of cancer. Abnormal copy number of G1-cell cycle related genes is frequently found in neuroblastoma and increased expression of E2F target genes [25]. Metastasis is a hallmark of malignant neuroblastoma and is the main reason for therapeutic failure. TNF-a is a proinflammatory cytokine that activates the nuclear factor-KB signaling pathway, leading to CXCR4 
overexpression, fostering neuroblastoma cell metastasis[26]. Thus, it is a plausible inference for the 16IncRNAs associated with survival of patients with neuroblastoma. The potential molecular function may put forward the direction for the further study on the mechanism of neuroblastoma progression.

There are some limitations in our study. We cannot exclude the possibility of residual confounding after internal validation as a result of possible overfitting from variable and threshold selection for these models. So, internal validation with bootstrapping and external validation were used to address these concerns. In addition, the underlying mechanisms of these IncRNAs in our risk score remain unclear. Further cell and animal studies are needed to confirm the exact molecular mechanisms of these IncRNAs.

In conclusion, this study identified a novel and robust IncRNA-based LASSO risk score for prognostic prediction of neuroblastoma by mining currently available microarray data. This risk score may contribute to personalize prediction of neuroblastoma prognosis and acted as potential biomarkers. A nomogram combining the molecular signature and clinical factors was constructed. The nomogram that contain the 16-IncRNAs allows for accurate risk assessment and guides future clinical planning regarding patient surveillance.

\section{Declarations}

\section{Acknowledgements}

Thanks for Gene Expression Omnibus (GEO) database(https://www.ncbi.nlm.nih.gov/geo/info/).

\section{Authors' contributions}

Yong Lv designed, analyzed the data, and write the manuscript.

Bo Xiang and ShuGuang Jin conceptualized and developed an outline for the manuscript and revised the manuscript.

\section{Funding}

1.3.5 project for disciplines of excellence, West China Hospital,Sichuan University”.邓ZYJC18003】

\section{Availability of data and materials}

The datasets used and/or analyzed during the current study are available from the corresponding author on reasonable request.

\section{Ethics approval and consent to participate}

The paper is exempt from ethical committee approval. Because no human specimens were used, and the data were download from public databases (GEO), which had been ethically reviewed.

\section{Consent for publication}


Yes.

\section{Competing interests}

There is no conflict of interest.

\section{References}

1. Wong M, Sun Y, Xi Z, Milazzo G, Poulos RC, Bartenhagen C, et al. JMJD6 is a tumorigenic factor and therapeutic target in neuroblastoma. Nat Commun. 2019 Jul 25;10(1):3319.

2. Pinto NR, Applebaum MA, Volchenboum SL, Matthay KK, London WB, Ambros PF, et al. Advances in Risk Classification and Treatment Strategies for Neuroblastoma. J Clin Oncol. 2015 Sep 20;33(27):3008-17.

3. Bagatell R, Cohn SL. Genetic discoveries and treatment advances in neuroblastoma. Curr Opin Pediatr. 2016 Feb;28(1):19-25.

4. Wang J, Wang Z, Yao W, Dong K, Zheng S, Li K. The association between IncRNA LINC01296 and the clinical characteristics in neuroblastoma. J Pediatr Surg. 2019 Dec;54(12):2589-94.

5. Qu L, Wang ZL, Chen Q, Li YM, He HW, Hsieh JJ, et al. Prognostic Value of a Long Non-coding RNA Signature in Localized Clear Cell Renal Cell Carcinoma. Eur Urol. 2018 Dec;74(6):756-63.

6. Zhu X, Tian X, Yu C, Shen C, Yan T, Hong J, et al. A long non-coding RNA signature to improve prognosis prediction of gastric cancer. Mol Cancer. 2016 Sep 20;15(1):60.

7. He A, He S, Peng D, Zhan Y, Li Y, Chen Z, et al. Prognostic value of long non-coding RNA signatures in bladder cancer. Aging (Albany NY). 2019 Aug 20;11(16):6237-51.

8. Yu Y, Chen F, Yang Y, Jin Y, Shi J, Han S, et al. IncRNA SNHG16 is associated with proliferation and poor prognosis of pediatric neuroblastoma. Int J Oncol. 2019 Jul;55(1):93-102.

9. Liu PY, Tee AE, Milazzo G, Hannan KM, Maag J, Mondal S, et al. The long noncoding RNA IncNB1 promotes tumorigenesis by interacting with ribosomal protein RPL35. Nat Commun. 2019 Nov 5;10(1):5026.

10. Zhao X, Li D, Huang D, Song H, Mei H, Fang E, et al. Risk-Associated Long Noncoding RNA FOXD3AS1 Inhibits Neuroblastoma Progression by Repressing PARP1-Mediated Activation of CTCF. Mol Ther. 2018 Mar 7;26(3):755-73.

11. Balachandran VP, Gonen M, Smith JJ, DeMatteo RP. Nomograms in oncology: more than meets the eye. Lancet Oncol. 2015 Apr;16(4):e173-80.

12. Peng F, Wang R, Zhang Y, Zhao Z, Zhou W, Chang Z, et al. Differential expression analysis at the individual level reveals a IncRNA prognostic signature for lung adenocarcinoma. Mol Cancer. 2017 Jun 6;16(1):98.

13. Tang J, Ren J, Cui Q, Zhang D, Kong D, Liao X, et al. A prognostic 10-IncRNA expression signature for predicting the risk of tumour recurrence in breast cancer patients. J Cell Mol Med. 2019 Oct;23(10):6775-84. 
14. Zhu X, Tian X, Sun T, Yu C, Cao Y, Yan T, et al. GeneExpressScore Signature: a robust prognostic and predictive classifier in gastric cancer. Mol Oncol. 2018 Nov;12(11):1871-83.

15. Fakhry C, Zhang Q, Nguyen-Tan PF, Rosenthal DI, Weber RS, Lambert L, et al. Development and Validation of Nomograms Predictive of Overall and Progression-Free Survival in Patients With Oropharyngeal Cancer. J Clin Oncol. 2017 Dec 20;35(36):4057-65.

16. Debrabant B. The null hypothesis of GSEA, and a novel statistical model for competitive gene set analysis. Bioinformatics. 2017 May 1;33(9):1271-77.

17. Liao Y, Wang X, Zhong P, Yin G, Fan X, Huang C. A nomogram for the prediction of overall survival in patients with stage II and III non-small cell lung cancer using a population-based study. Oncol Lett. 2019 Dec;18(6):5905-16.

18. Wang Z, Gao L, Guo X, Feng C, Lian W, Deng K, et al. Development and validation of a nomogram with an autophagy-related gene signature for predicting survival in patients with glioblastoma. Aging (Albany NY). 2019 Dec 17;11(24):12246-69.

19. Ouyang D, Li R, Li Y, Zhu X. A 7-IncRNA signature predict prognosis of Uterine corpus endometrial carcinoma. J Cell Biochem. 2019 Oct;120(10):18465-77.

20. Ma B, Li Y, Ren Y. Identification of a 6-IncRNA prognostic signature based on microarray reannotation in gastric cancer. Cancer Med. 2020 Jan;9(1):335-49.

21. Gao YF, Liu JY, Mao XY, He ZW, Zhu T, Wang ZB, et al. LncRNA FOXD1-AS1 acts as a potential oncogenic biomarker in glioma. CNS Neurosci Ther. 2020 Jan;26(1):66-75.

22. Li SY, Wang H, Mai HF, Li GF, Chen SJ, Li GS, et al. Down-regulated long non-coding RNA RNAZFHX4AS1 suppresses invasion and migration of breast cancer cells via FAT4-dependent Hippo signaling pathway. Cancer Gene Ther. 2019 Nov;26(11-12):374-87.

23. Liu Q, Wu Y, Xiao J, Zou J. Long Non-Coding RNA Prostate Cancer-Associated Transcript 7 (PCAT7) Induces Poor Prognosis and Promotes Tumorigenesis by Inhibiting mir-134-5p in Non-Small-Cell Lung (NSCLC). Med Sci Monit. 2017 Dec 24;23:6089-98.

24. Schramm A, Koster J, Assenov Y, Althoff K, Peifer M, Mahlow E, et al. Mutational dynamics between primary and relapse neuroblastomas. Nat Genet. 2015 Aug;47(8):872-7.

25. Molenaar JJ, Koster J, Ebus ME, van Sluis P, Westerhout EM, de Preter K, et al. Copy number defects of G1-cell cycle genes in neuroblastoma are frequent and correlate with high expression of E2F target genes and a poor prognosis. Genes Chromosomes Cancer. 2012 Jan;51(1):10-9.

26. Zhi Y, Lu H, Duan Y, Sun W, Guan G, Dong Q, et al. Involvement of the nuclear factor-kappaB signaling pathway in the regulation of CXC chemokine receptor-4 expression in neuroblastoma cells induced by tumor necrosis factor-alpha. Int J Mol Med. 2015 Feb;35(2):349-57.

\section{Tables}

Due to technical limitations, tables are only available as a download in the Supplemental Files section. 


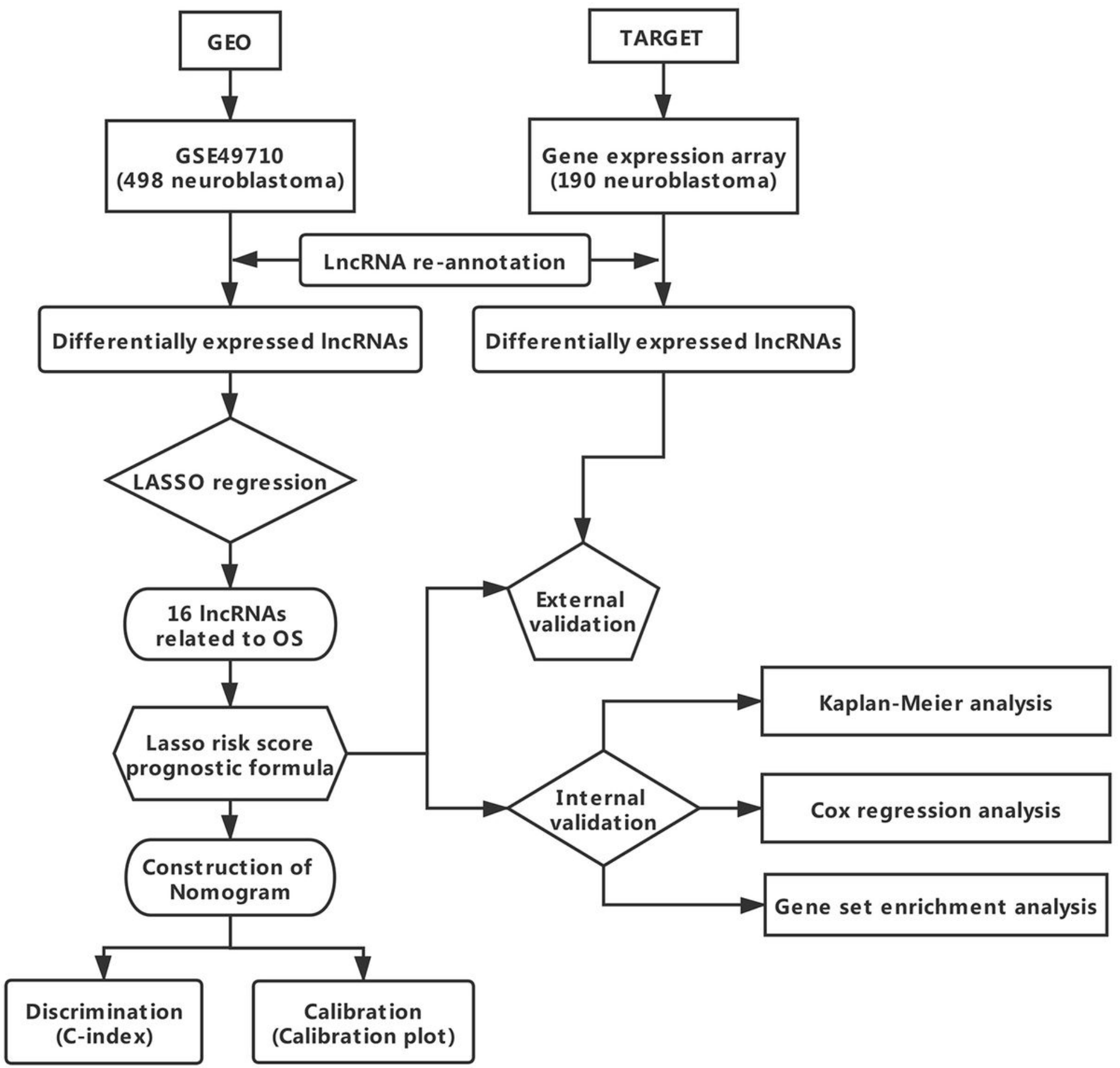

Figure 1

The flowchart of this study 

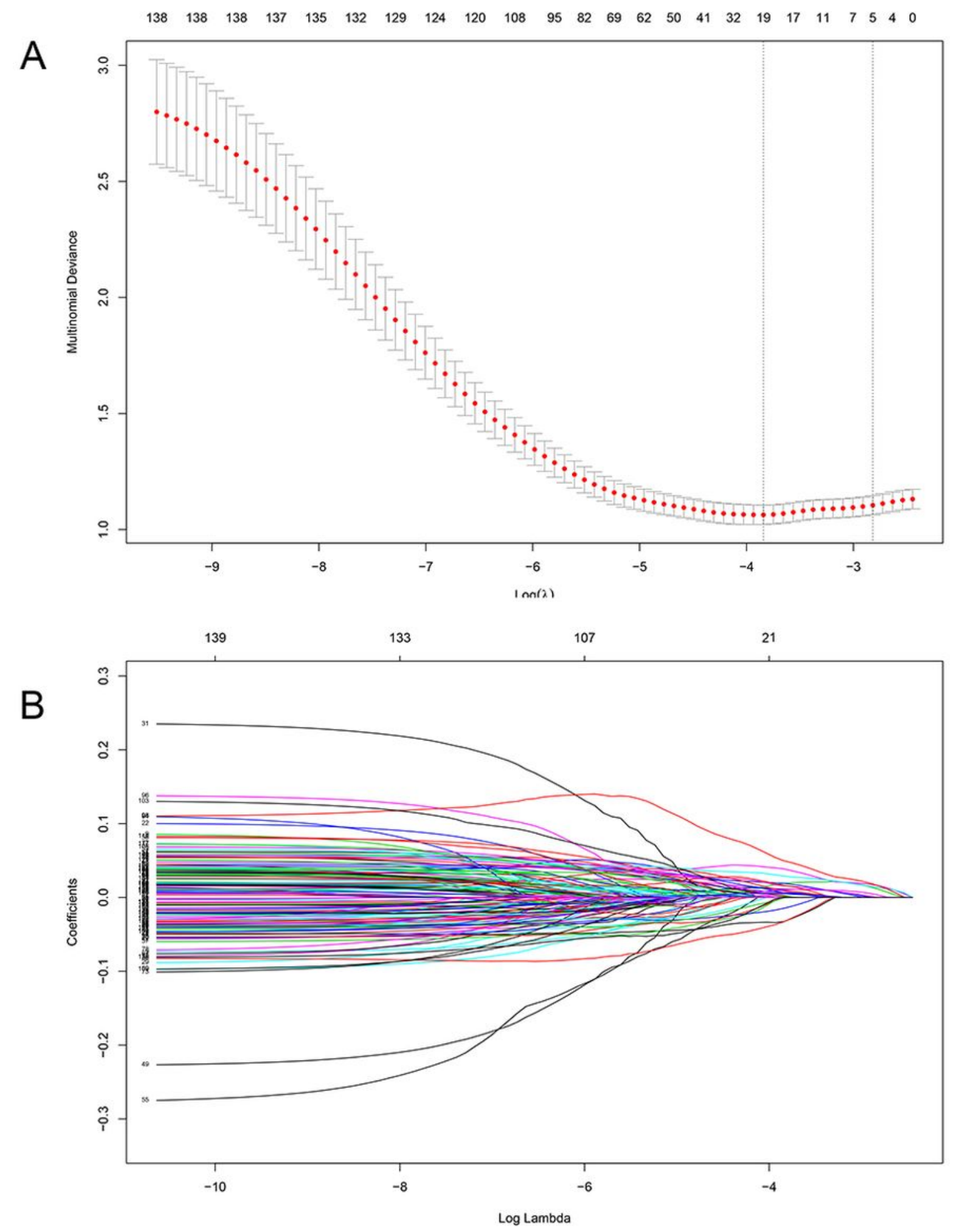

\section{Figure 2}

Prognostic IncRNAs selection using the least absolute shrinkage and selection operator (LASSO) regression algorithm. (A) Tuning parameter $(\lambda)$ selection in the LASSO model used 10 -fold crossvalidation via minimum criteria. (B) LASSO coefficient profiles of the 151 IncRNAs. 

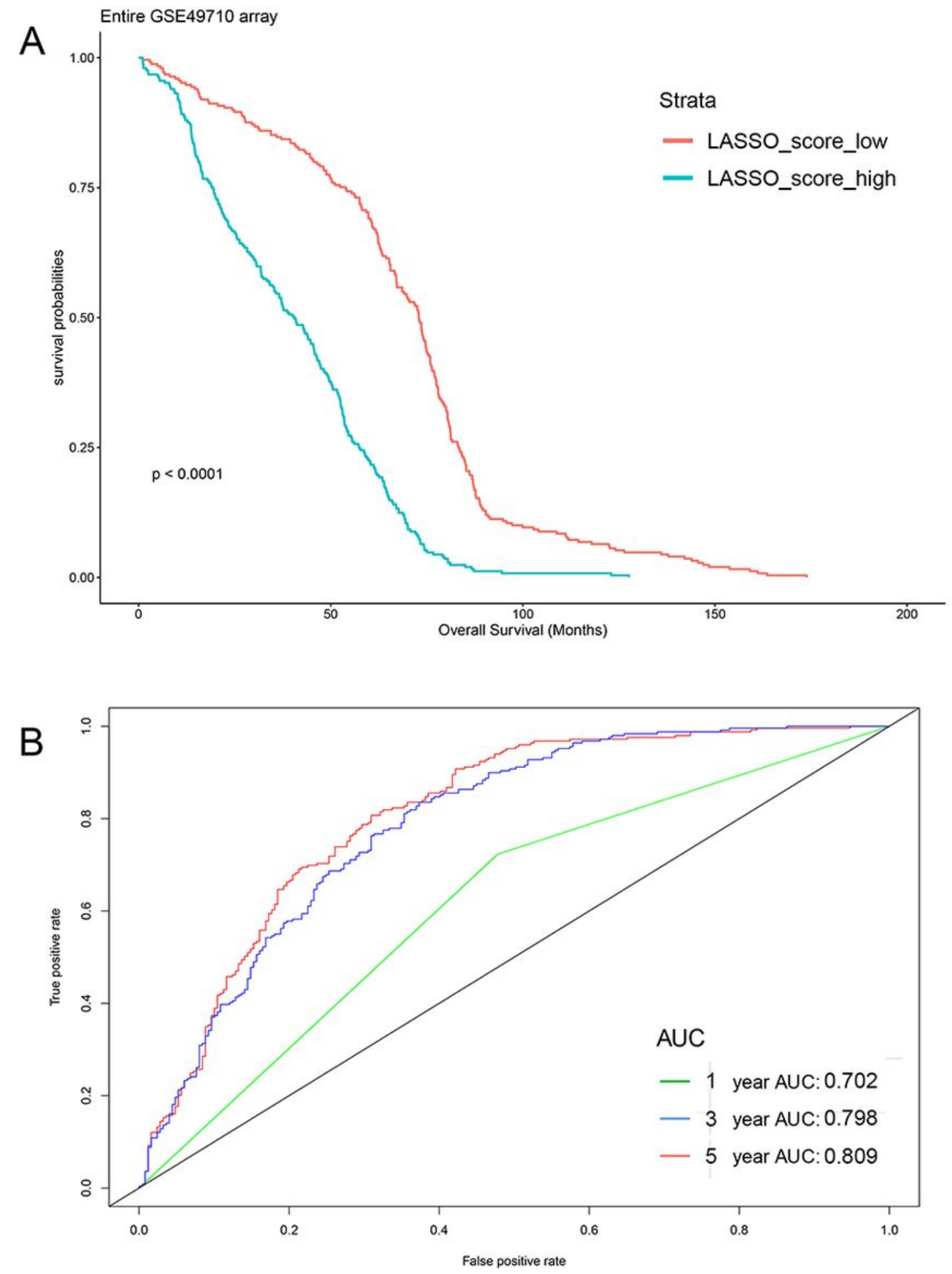

\section{Figure 3}

Internal validation of the prognostic risk score in GSE49710 series. (A) Kaplan-Meier survival analysis of the 16-IncRNA-based LASSO risk score. (B) Time-dependent ROC curves of the 16-IncRNA-based LASSO risk score. 

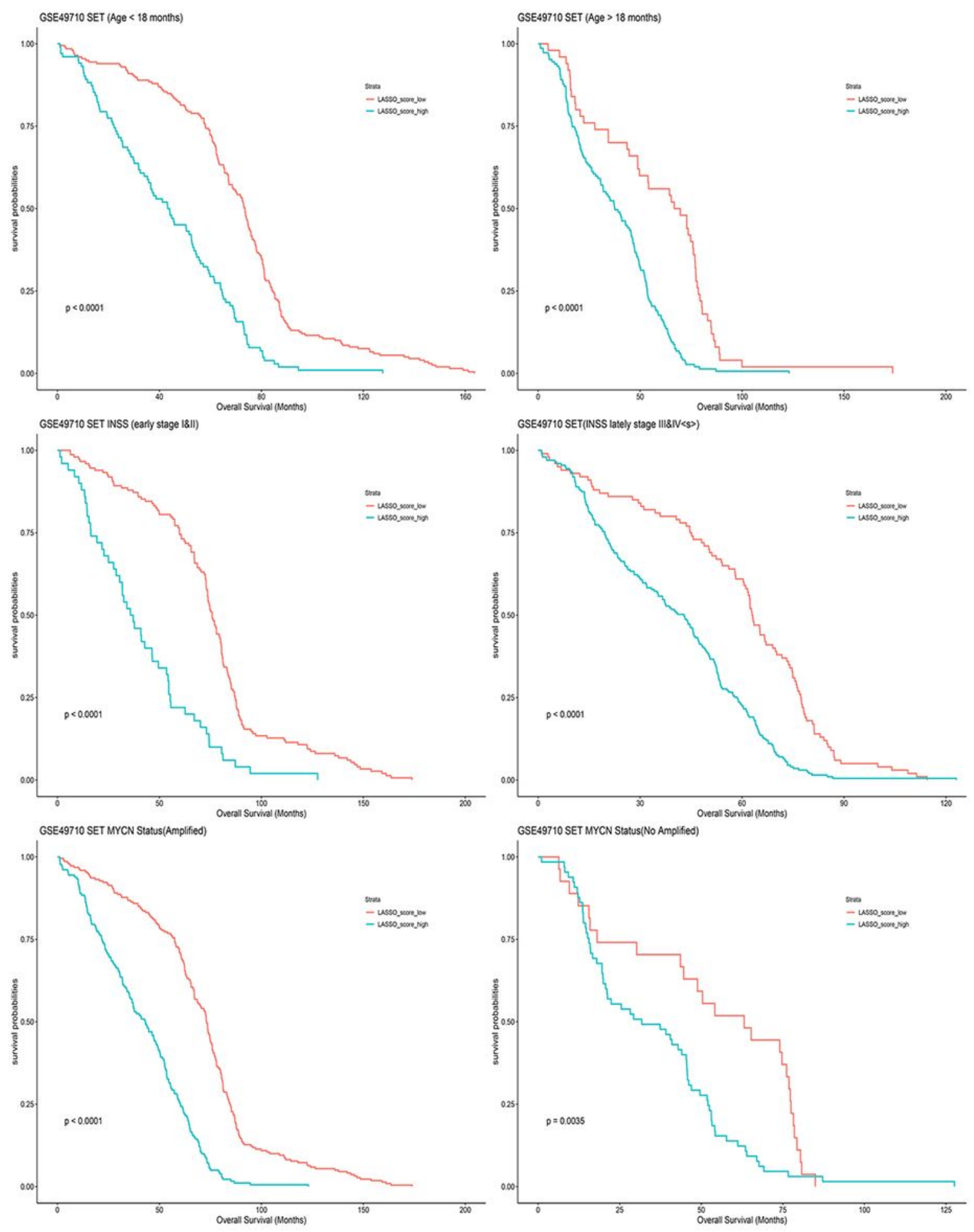

Figure 4

Kaplan-Meier estimates of the overall survival of GSE49710 series using the LASSO risk score, stratified by age, INSS stage and MYCN status. 

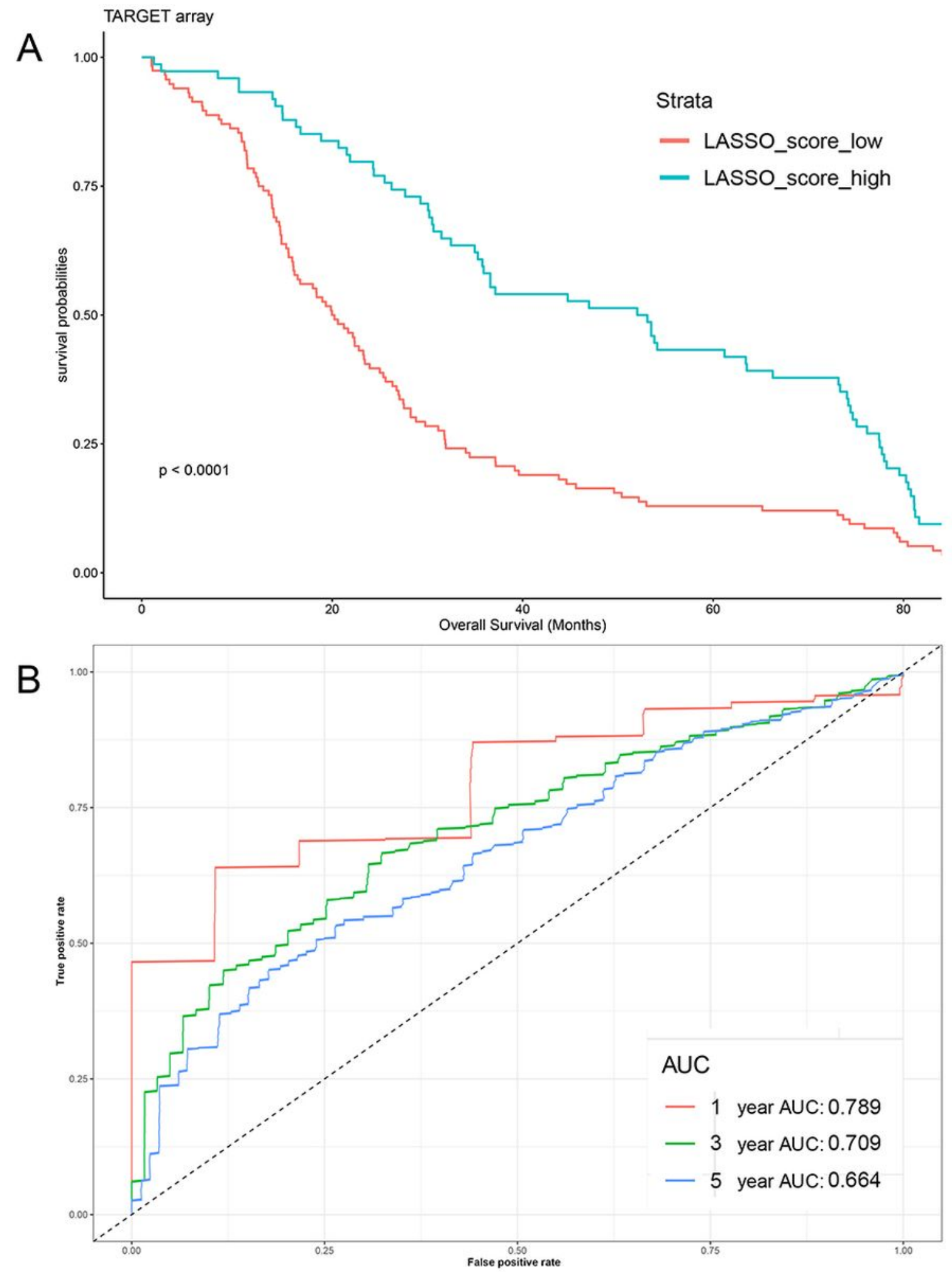

\section{Figure 5}

External validation of the prognostic risk score in TARGET series. (A) Kaplan-Meier survival analysis of the 16-IncRNA-based LASSO risk score. (B) Time-dependent ROC curves of the 16-IncRNA-based LASSO risk score. 


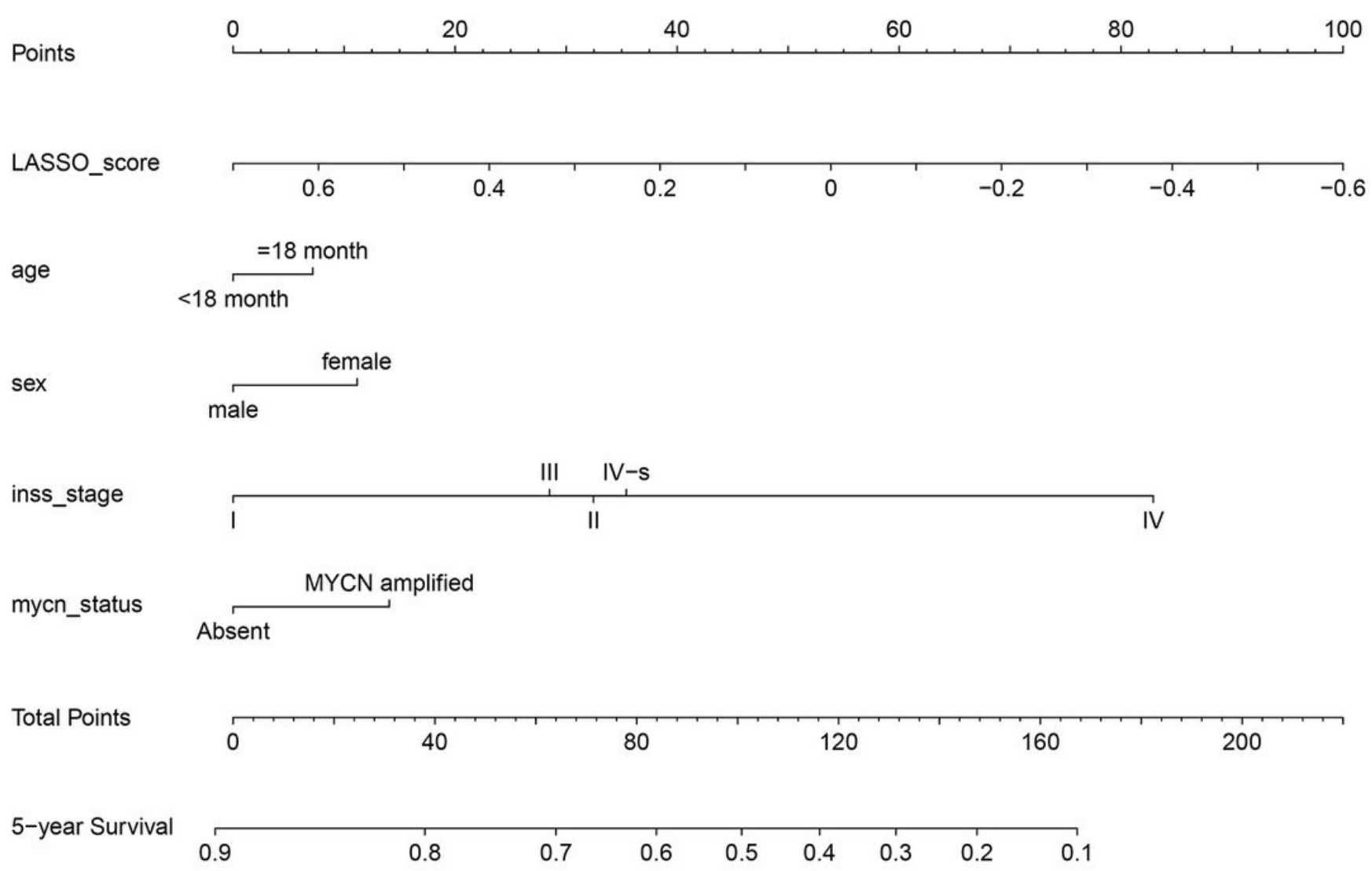

\section{Figure 6}

Nomogram to predict 5-year overall survival probability in neuroblastoma patients 

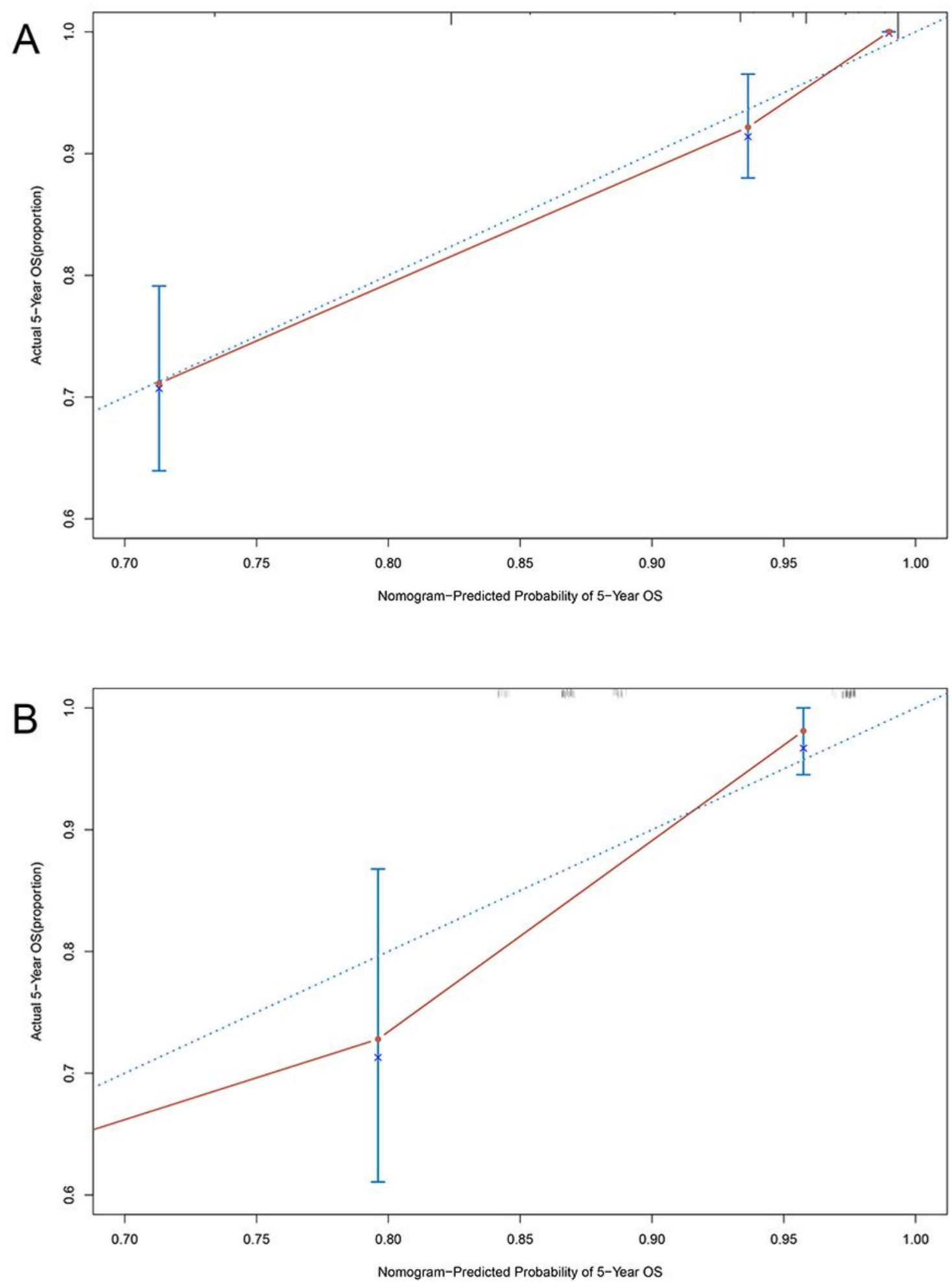

Figure 7

Calibration plots of the nomogram (A) Nomogram-predicted probability of 5-year overall survival in GSE49710 (B) Nomogram-predicted probability of 5-year overall survival in TARGET 
Enrichment plot: HALLMARK_E2F_TARGETS

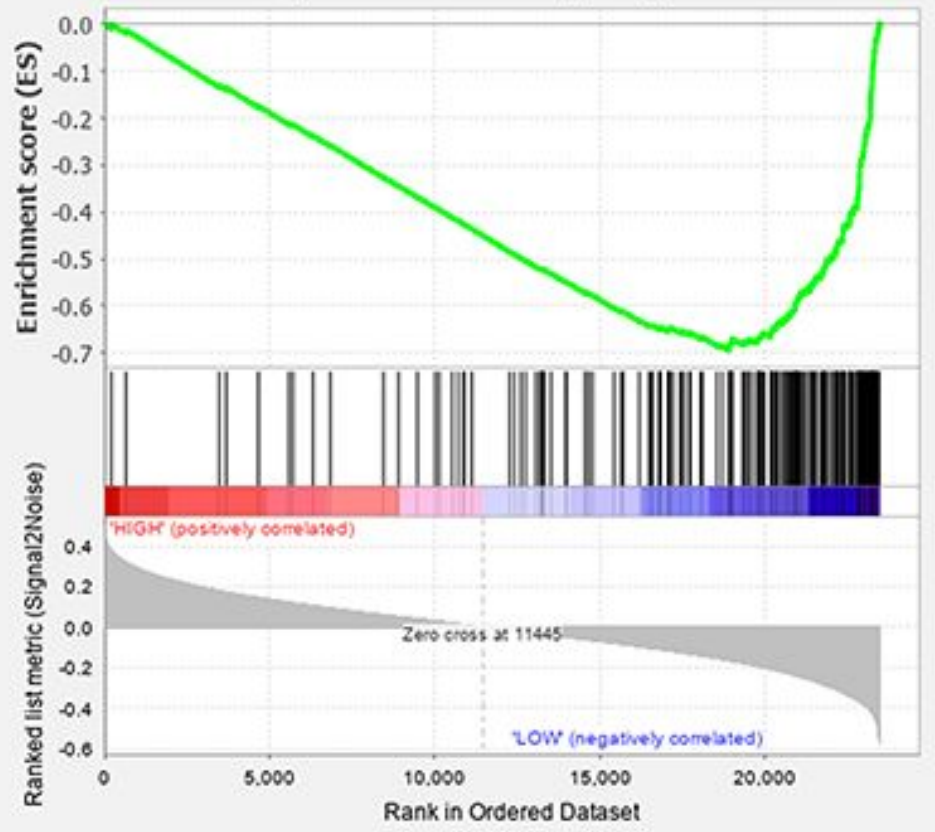

- Enrichment profile - Hits — Ranking metric scores

Enrichment plot:

HALLMARK_INFLAMMATORY_RESPONSE
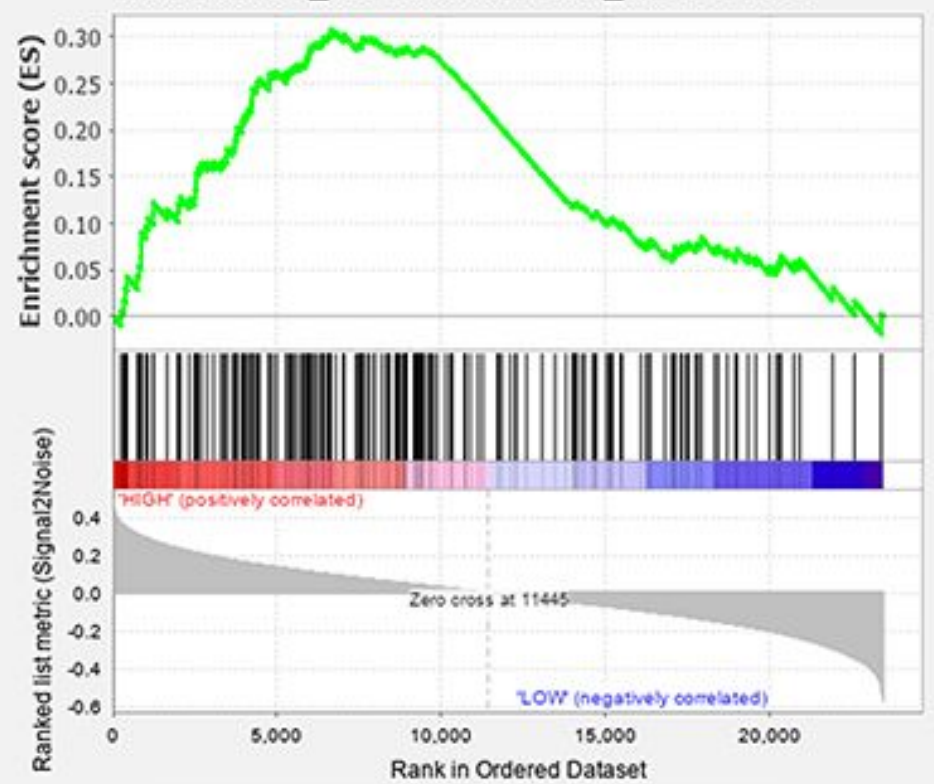

Enrichment profile - Hits

Ranking metric scores
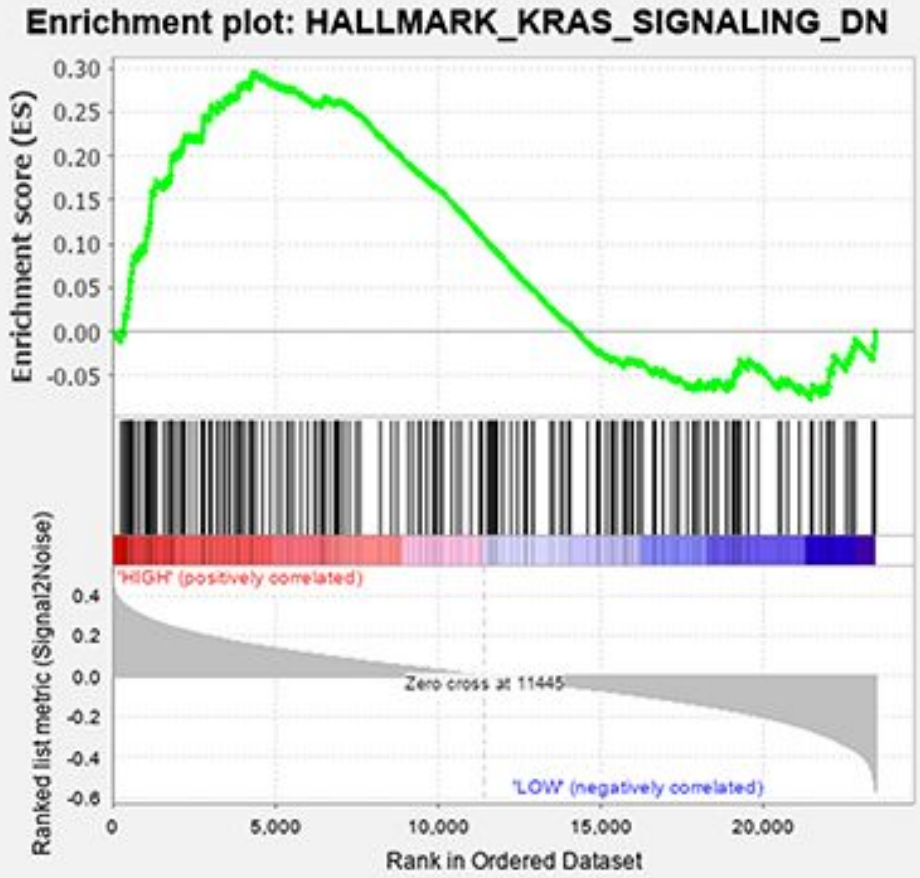

Enrichment profile - Hits - Ranking metric scores

Enrichment plot:

HALLMARK_TNFA_SIGNALING_VIA_NFKB
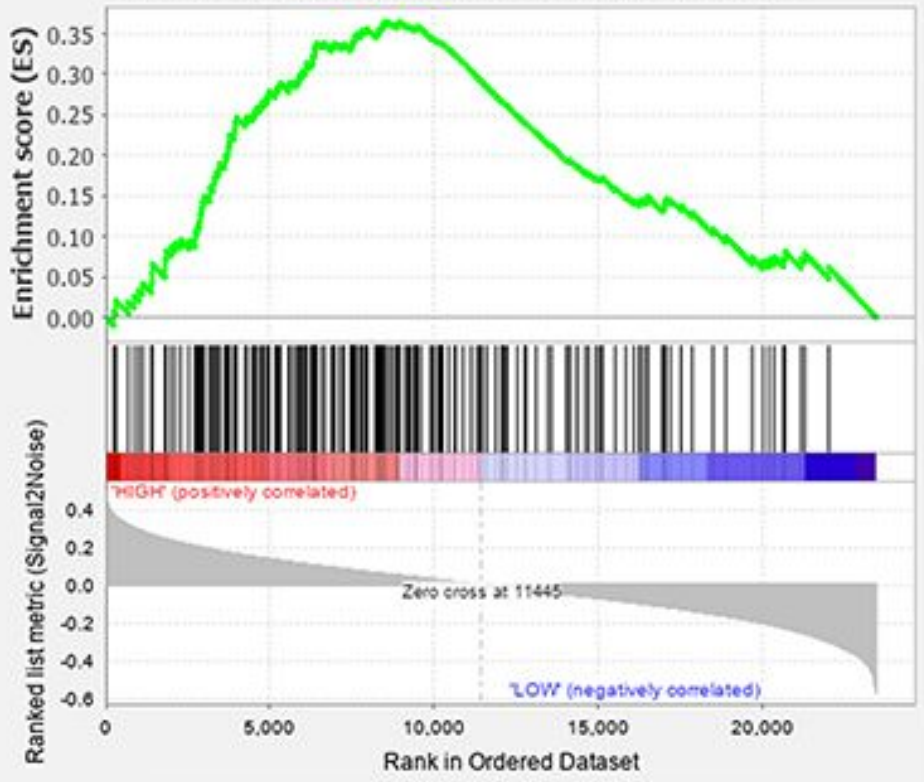

Enrichment profile - Hits — Ranking metric scores

Figure 8

Gene set enrichment analysis delineates biological pathways correlated with 16-IncRNAs

\section{Supplementary Files}

This is a list of supplementary files associated with this preprint. Click to download. 
- Table1.xIsx

- Table2.xIsx

- Table3.xIsx

Page 19/19 